\title{
THE USE OF A DEBUNCHER IN THE INJECTION PATH OF AN ELECTRON SYNCHROTRON
}

\author{
by
}

M. C. Crowley-Milling

and

G. Saxon 
CONTENTS

Page

1. INTRODUCTION I

2. CORRUGATED WAVEGUIDE DESIGN 2

3. BEAM LOADING CONSIDERATIONS 4

4. DESCRIPTION OF THE WAVEGUIDE ASSEMBLY 5

5. THE WAVEGUIDE FEED SYSTEM 5

$\begin{array}{ll}\text { 6. OPERATIONAL TESTS } & 10\end{array}$

7. CONCLUSIONS 12

$\begin{array}{ll}\text { REFERENCES } & 12\end{array}$ 
The principle of reducing the energy spread in a bunched electron beam by debunching and using a correcting r.f. cavity was first proposed a long time ago ${ }^{(1)}$, but the system described in this paper is believed to be the first application. Unlike the case for proton beams, where debunching in a drift. space occurs due to the velocity variation, a magnetic system which provides a dispersion in path length proportional to energy error must be provided. In the origina? design of the electron synchrotron NINA, provision was made for the injection of positrons, and to avoid difficulties with the positron system when injecting a large current of electrons, a magnetic bypass system was installed to take the electrons round the positron converter and accelerator as shown in fig. 1. The ends of the pole pieces of the bending magnets are cut at

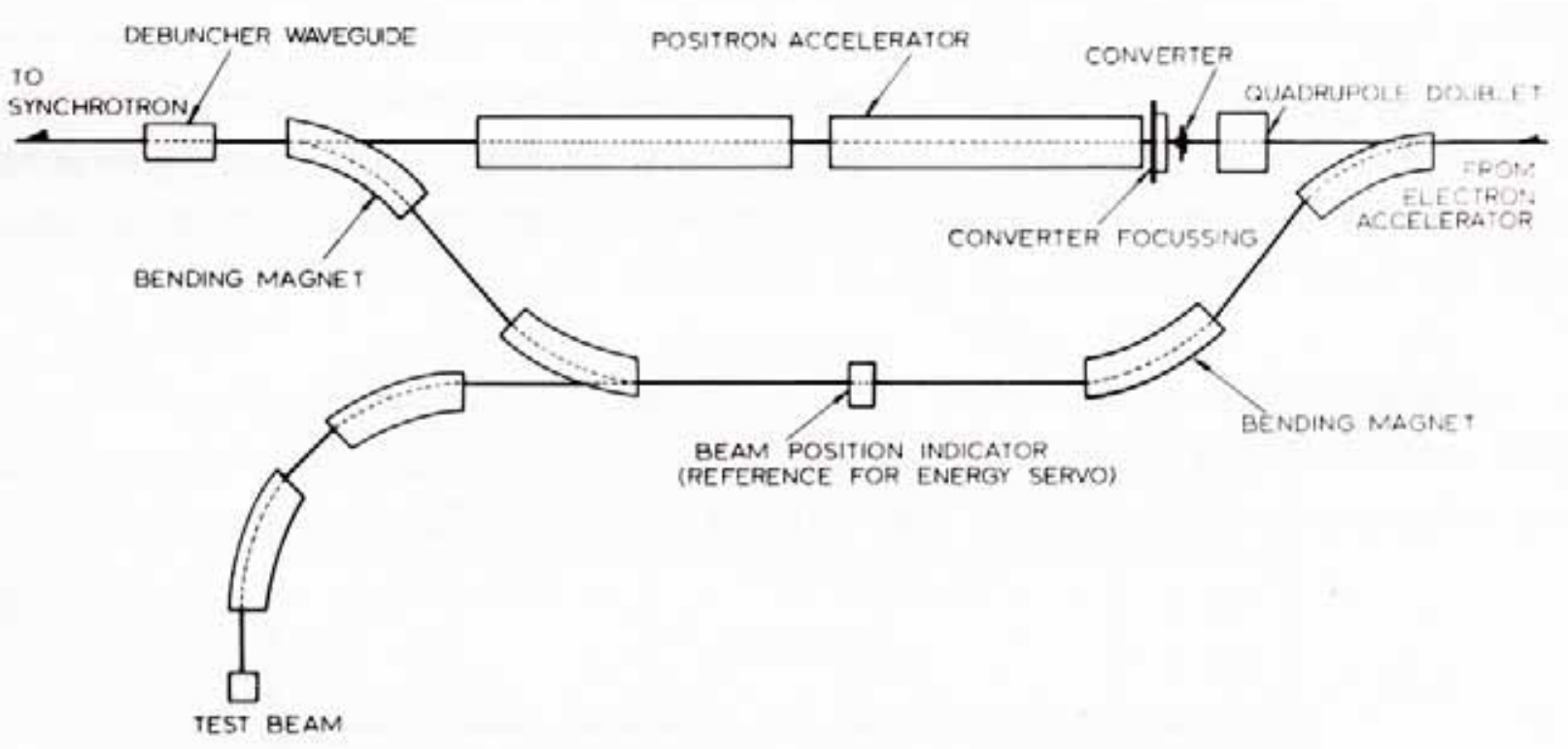

Fig. 1 Layout of bypass system for NINA injector.

the appropriate angle to provide a unity transfer matrix from one end of the bypass to the other, so the system is achromatic, but it is not isochronous, giving different path lengths for electrons of differing momenta. This results 
in a tightly-bunched beam of electrons becoming partly debunched, and to reduce the energy spread in the beam, a short length of linear accelerator waveguide is inserted in the path of the beam after the bypass. This waveguide is supplied with $r . f$. power at the same frequency as the linear accelerator. The power level and phasing are adjusted so that high energy electrons lose momentum whilst those of energy lower than the correct value gain momentum. In this way an initial energy spread in the beam of $\pm 3 \%$ can be reduced by a factor of about 10 .

\section{CORRUGATED WAVEGUIDE DESIGN}

Calculations on the bypass system showed that the path difference for a $1 \%$ momentum change is $13.6 \mathrm{~mm}$, corresponding to a phase difference of $47^{\circ}$ at 2856 $\mathrm{MHz}$. Neglecting beam loading effects and considering a $43 \mathrm{MeV}$ electron beam, a particle off momentum by $1 \%$ must be made to gain or lose $0.43 \mathrm{MeV}$ in traversing the short length of corrugated waveguide when riding at $47^{\circ}$ with respect to the zero of the wave. In the ideal case, in which the bunch phase width in the incoming beam is negligible, the energy error would be reduced in the manner shown in fig. 2(a). However, the NINA injector is designed for a high beam current, and space charge effects during bunching result in bunches of appreciable phase width, between 5 and $10^{\circ}$. However, a worthwhile improvement can be obtained even for a $10^{\circ}$ bunch width, as can be seen from fig. 2(b).

The properties of waveguide with 3 corrugations per wavelength were derived from available data ${ }^{(2)}$ and are summarised in Table 1 . The series impedance is the relationship between power flow $(W)$ and peak field $(E)$ given by $E^{2} / W$.

\section{TABLE 1}

\begin{tabular}{|c|c|c|}
\hline $\begin{array}{c}\text { Iris hole radius } \\
(\mathrm{mm})\end{array}$ & $\begin{array}{c}\text { Series Impedance } \\
\left(\mathrm{M} \Omega / \mathrm{m}^{2}\right)\end{array}$ & $\begin{array}{c}\text { Attenuation } \\
(\mathrm{db} / \mathrm{m})\end{array}$ \\
\hline 11 & 19.4 & 1.5 \\
12 & 14.2 & 1.13 \\
13.5 & 8.7 & 0.71 \\
15 & 6.1 & 0.54 \\
\hline
\end{tabular}



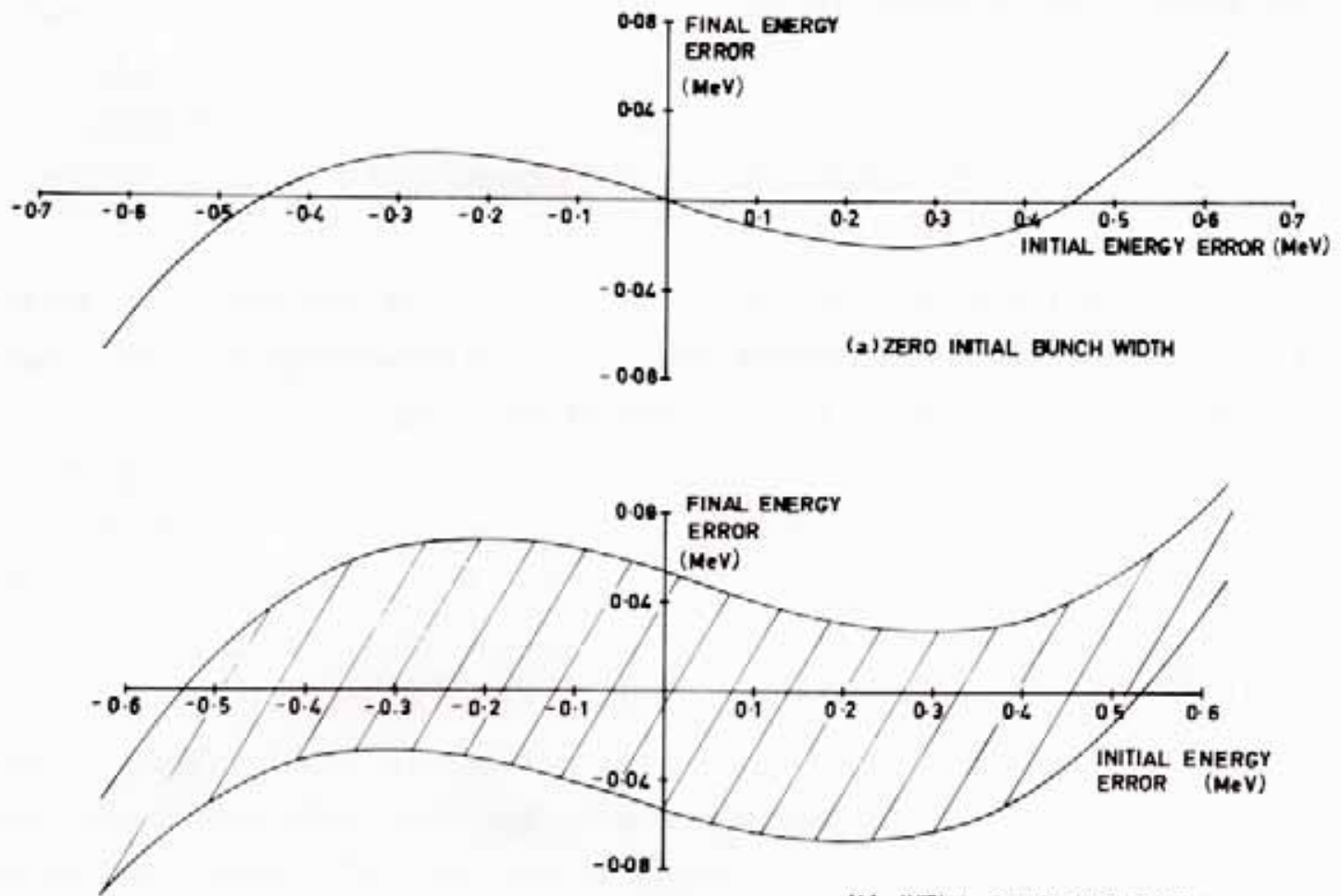

(b) INITIAL BUNCH WIDTH 10,

Fig. 2 Reduction of energy error by debuncher system.

For the short lengths of waveguide under consideration attenuation effects may be neglected. Table 2 shows the lengths of waveguide needed, with $0.5 \mathrm{MW}$ power input, to correct a $1 \%$ energy error at a phase angle of $47^{\circ}$.

TABLE 2

\begin{tabular}{|c|c|c|}
\hline $\begin{array}{c}\text { Iris hole radius } \\
(\mathrm{mm})\end{array}$ & $\begin{array}{c}\text { Peak field for 0.5MW } \\
(\mathrm{MV} / \mathrm{m})\end{array}$ & $\begin{array}{c}\text { Length } \\
(\mathrm{m})\end{array}$ \\
\hline 11 & 3.20 & 0.194 \\
12 & 2.66 & 0.233 \\
13.5 & 2.08 & 0.297 \\
15 & 1.75 & 0.355 \\
\hline
\end{tabular}

Since the waveguide must be made of an integral number of corrugations, a convenient length was chosen of $0.28 \mathrm{~m}$ ( 8 corrugations) for which the iris hole radius is about $13.1 \mathrm{~mm}$. The reason for the choice of $0.5 \mathrm{MW}$ for the input power was that this could be conveniently obtained via a directional coupler from one of the high power feeder waveguides to the linear accelerator, in 
which the power flow is about $10 \mathrm{MW}$.

\section{BEAM LOADING CONSIDERATIONS}

The above considerations are modified slightly by the loading effects of a bunched beam on the corrugated waveguide. The field seen by a tight bunch riding at phase $\phi$ with respect to the zero of the wave is

$$
E_{z}=E_{0} e^{-\alpha z} \sin \phi-\frac{\xi I}{2} \frac{1-e^{-\alpha z}}{\alpha}
$$

where $E_{0}$ is the initial field $\left(\sqrt{\varepsilon W_{0}}\right)$. Neglecting the attenuation, $\alpha$,

$$
E_{z}=\sqrt{\xi W_{0}} \sin \phi-\xi \mathrm{Iz} / 2
$$

and the energy gain or loss, $V$, in length, L

$$
V=\sqrt{\xi W_{0} L} \sin \phi-\xi \mathrm{IL}^{2} / 4
$$

In order to make this zero the phase must be chosen so that

$$
\sin \phi=\frac{\sqrt{\xi} I L}{4 \sqrt{W_{0}}}
$$

For the chosen parameters and a current of $0.2 \mathrm{~A}, \sin \phi=0.063$ and $\phi=3.6^{\circ}$. In fact, there is not a tight bunch at this point and electrons are spread over a phase range of $2 \theta$ about the phase of $\phi$. The field generated by the beam is modified by a factor $\sin \theta / \theta$, so that

$$
V=\sqrt{\xi W_{0} L} \sin \phi-\frac{\xi I L^{2}}{4} \frac{\sin \theta}{\theta}
$$

For this to be zero $\sin \phi=\frac{\sqrt{\xi} \mathrm{I} L}{4 \sqrt{W_{0}}} \frac{\sin \theta}{\theta}$ 
For $\theta=47^{\circ} \sin \theta / \theta=0.89$ so that $\sin \theta=0.056$ and $\phi=3.2^{\circ}$ for the case of $I=0.2$. It is evident from the above that beam loading effects are small for the curents normally injected into the synchrotron and that, in any case, they may be compensated for by an appropriate phase change.

\section{DESCRIPTION OF THE WAVEGUIDE ASSEMBLY}

The corrugated waveguide assembly in its vacuum housing is illustrated in fig. 3, whilst fig. 4 is a photograph of the assembly in position in the flight path. The design and manufacture were carried out by Vickers Engineering. The electroformed waveguide is mounted between mode transformers, of the type usually employed in linear accelerators, which are supported from two end plates. These plates are themselves supported from a pivoted base plate. Stainless steel bellows permit movement of the assembly whilst maintaining the vacuum. This method of support is provided so that the corrugated waveguide may be swung out of the beam line, this being necessary if a positron beam is to be accelerated when a larger aperture is needed than is given by the iris hole in the correcting cavity. The movement of the waveguide is effected by means of a handwheel below the vacuum housing.

Because of the requirement to move the corrugated waveguide, the flanges in the rectangular waveguide through which $r$.f. power is fed into and out of the cavity are of the non-contacting type, a choke and a plain flange being used with a small separation. In order to minimise this separation and so reduce $r . f$. leakage the faces of the plain flanges are radiused.

Ceramic vacuum windows are used in the fixed rectangular waveguides where they are brought through the wall of the vacuum housing.

\section{THE WAVEGUIDE FEED SYSTEM}

The power required for the debuncher correcting cavity is coupled out from the waveguide feeder to the second section of the $43 \mathrm{MeV}$ electron linear accelerator. Under normal running conditions the power in this waveguide is $10 \mathrm{MW}$ so that a $10 \mathrm{db}$ coupler provides more than enough power for the debuncher 

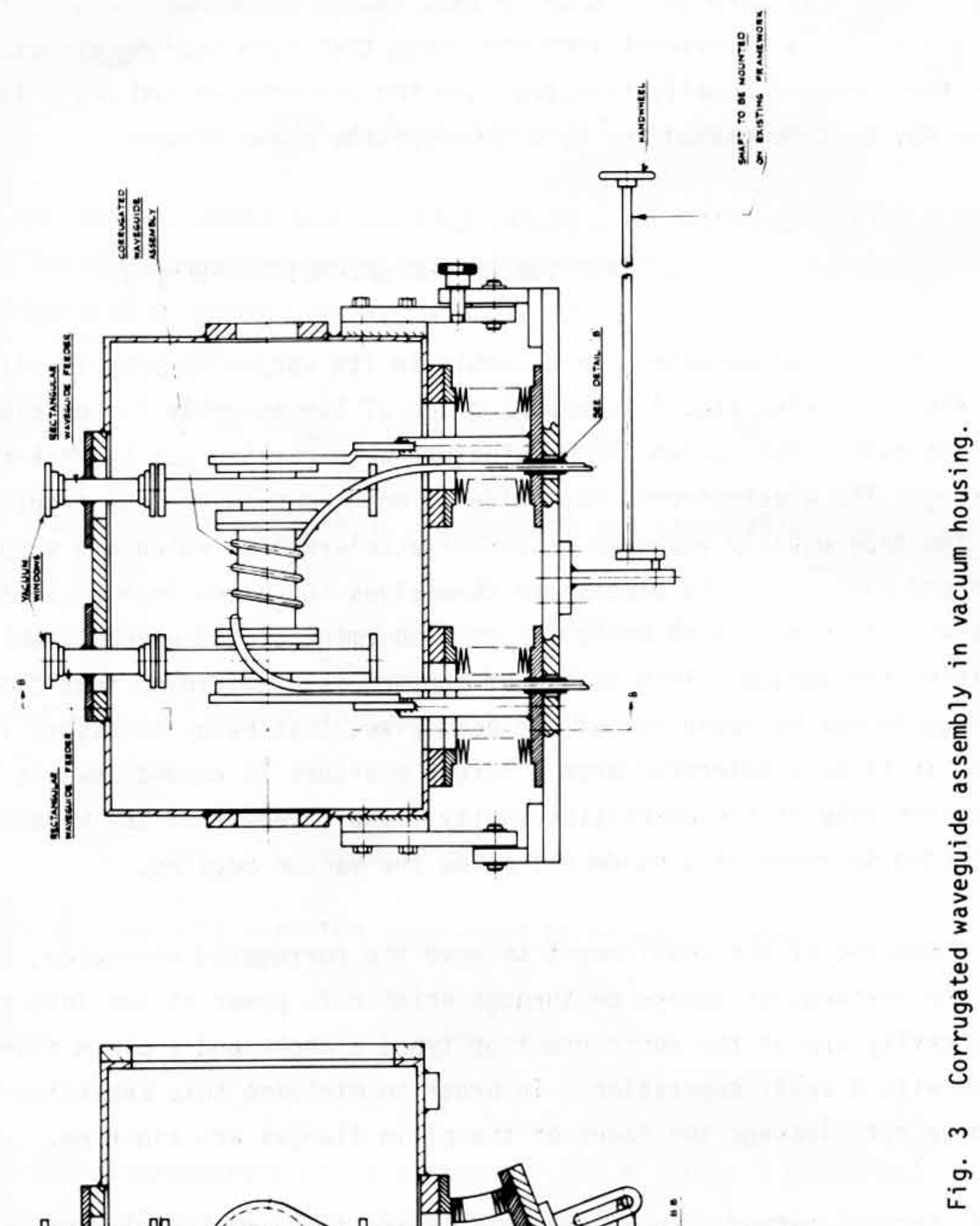

it 


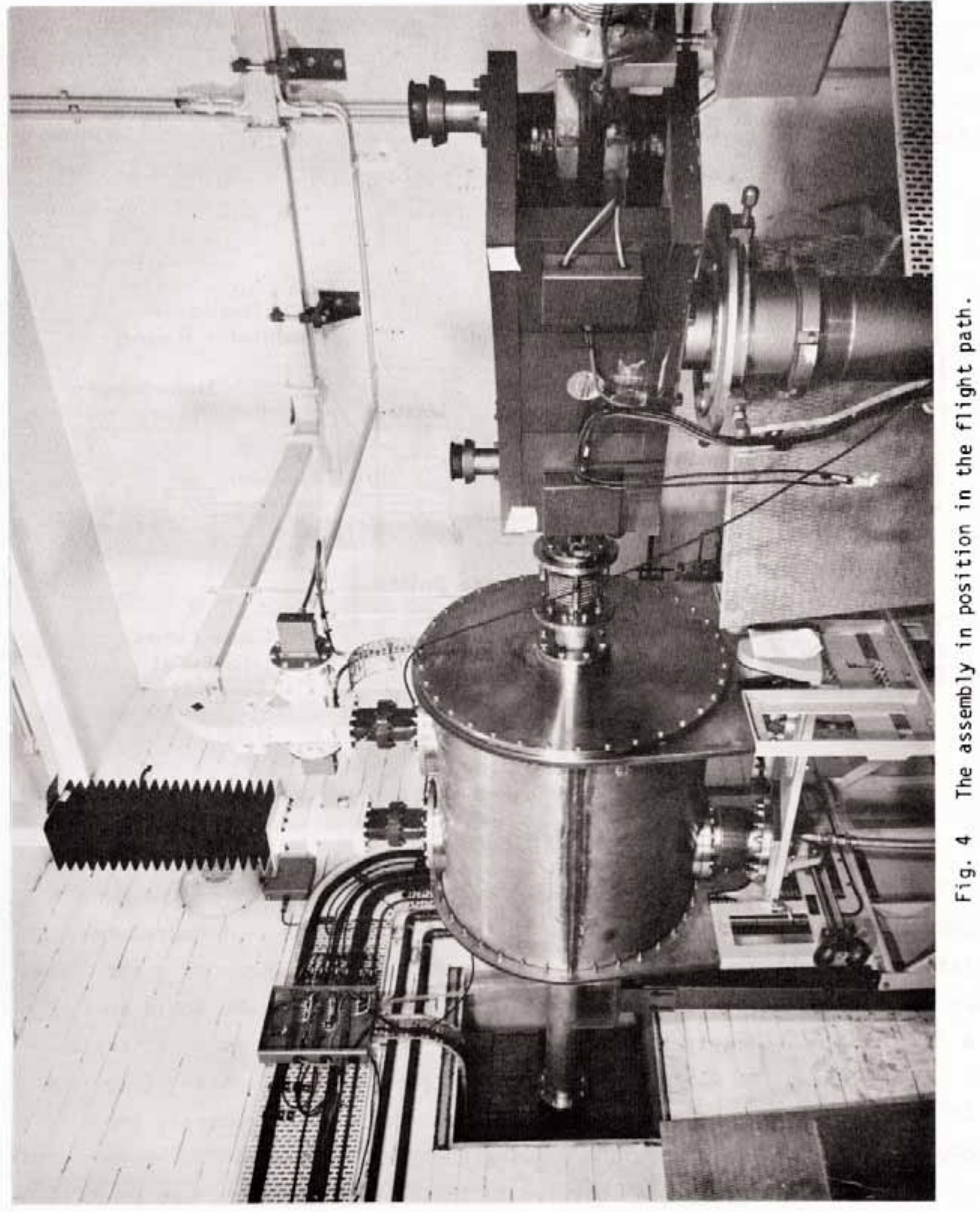


cavity. A variable power divider and a phase shifter are used to set the power level and phase to the correct values. An isolator in the waveguide feeder following the directional coupler minimises reaction back on the kiystron of reflections arising in the feeder system due to imperfections in its components. The r.f. system is shown schematically in fig. 5 and fig. 6 is a photograph showing the directional coupler, isolator, power divider and phase shifter mounted on the wall of the injector room.

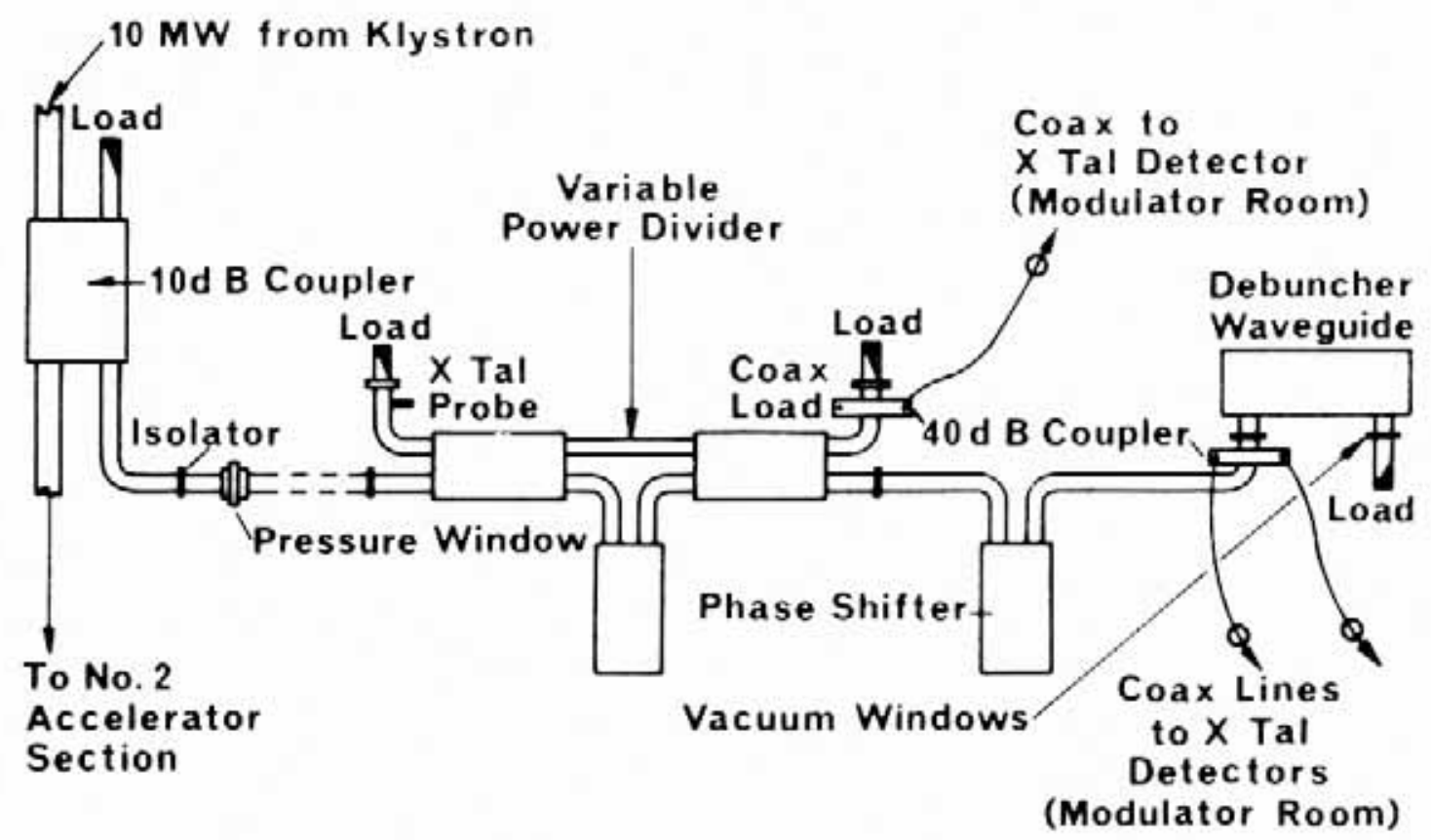

Fig. 5 Schematic arrangement of the r.f. system.

The distance from the directional coupler to the position of the debuncher is $21 \mathrm{~m}$. Dimensional changes in the waveguide due to temperature variation affect both the propagating wavelength and the length of guide. For copper waveguide the phase change per $1^{\circ} \mathrm{C}$ change of temperature would be $1.7^{\circ}$ in a $21 \mathrm{~m}$ length. Clearly temperature control of the feeder is necessary and this is done using the water system which controls the accelerator waveguides at $40^{\circ} \mathrm{C}$ and, since the debuncher waveguide requires similar control, the supply for the latter is run along pipes attached to the feeder waveguide, which is also well lagged. In addition to these precautions a phase servo system has been established which compares the phase of the supply at the cavity with a reference, and restores by driving the high power phase shifter. 


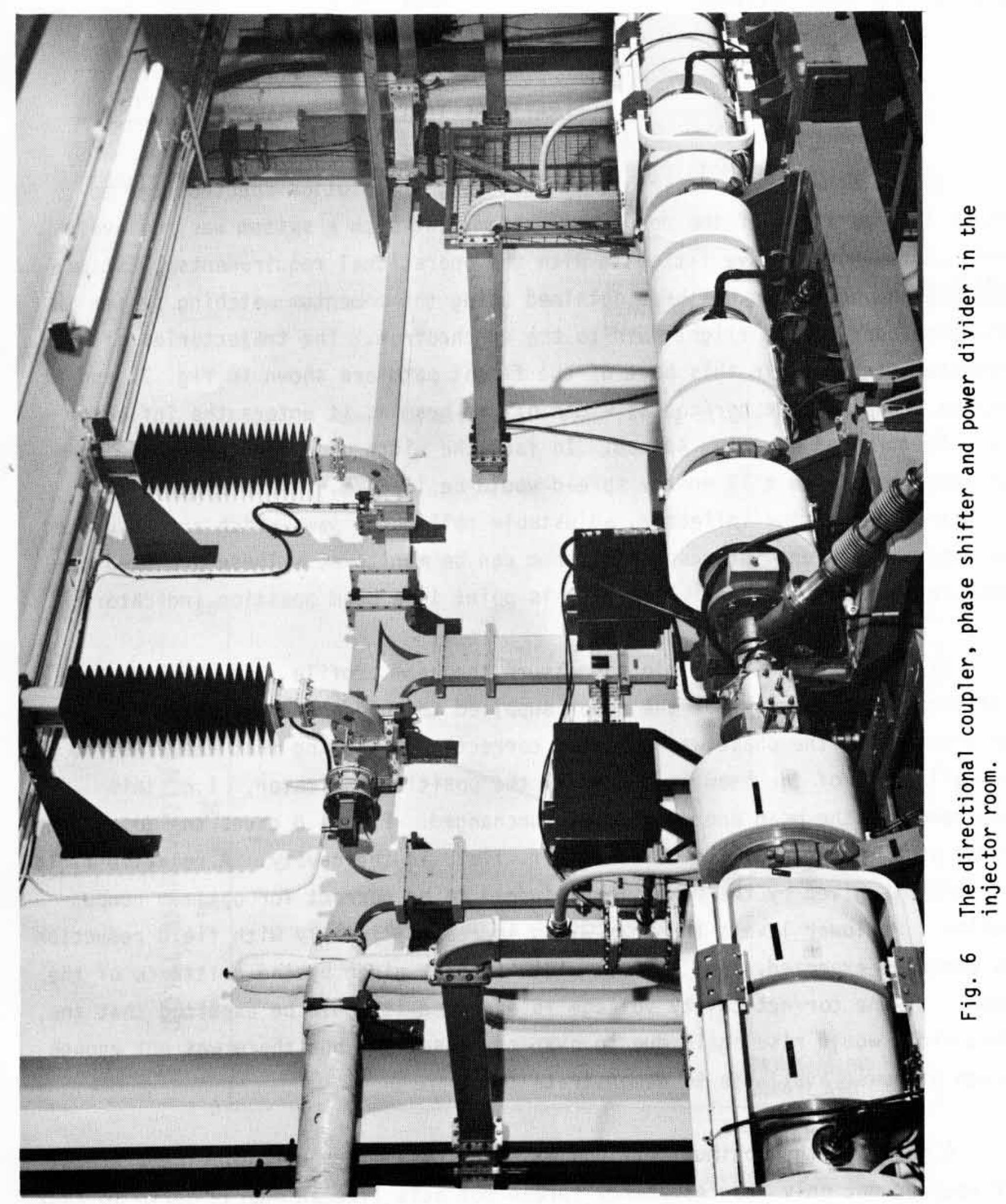


The control panel provides monitoring of the power divider and phase shifter settings, the phase error and the relative power in the two output arms of the power divider.

\section{OPERATIONAL TESTS}

It would have been desirable to use a high resolution spectrometer to check the operation of the debuncher system, but such a system was not available, nor would it have fitted in with the operational requirements. Estimation of the operation has been obtained using the momentum matching system in the remainder of the flight path to the synchrotron. The trajectories of offmomentum particles in this part of the flight path are shown in fig. 7 , and it may be seen that the horizontal width of the beam as it enters the inflector is a function of momentum spread. In fact the width of a zero emittance beam at this point with a $1 \%$ energy spread would be $13.8 \mathrm{~mm}$. There are, in the flight path near the inflector, adjustable collimator jaws which are insulated so that the current intercepted by them can be monitored. These are used to measure the beam profile. Also at this point is a beam position indicator.

It was therefore possible to measure the beam profile just prior to the inflector as a function of the power supplied to the debuncher cavity and also to ensure that the phase was adjusted correctly by showing that there was no overall shift of the beam as judged by the position indicator, i.e. this ensures that the mean energy remained unchanged. Figure 8 gives the total beam width as a function of the relative $r$.f. field in the cavity. A relative field of unity is given by the power level judged to be correct for optimum compensation. At lower levels the beam width increases linearly with field reduction as would be expected. The minimum width is that given by the emittance of the beam. If the correct cavity voltage is exceeded it would be expected that the beam width would rise again due to over-compensation, but there was not enough range of power available to demonstrate this.

A check on the optimum settings was made by another method. The system, of course, not only reduces energy spread but acts like an energy servo so that departures from the correct energy at the linear accelerator output are corrected by the debuncher cavity. The linear accelerator energy was deliberately varied by $\pm 0.5 \%$ and observation made of the position of the beam at 


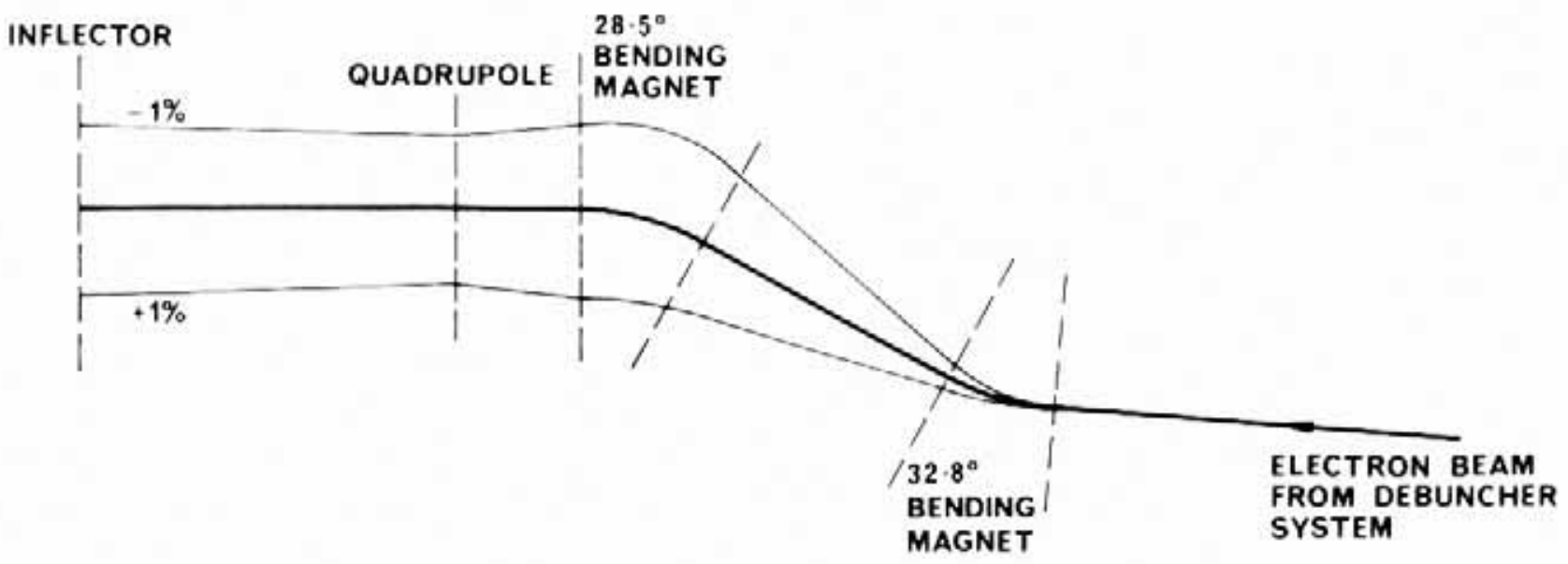

Fig. 7 Momentum matching system in the flight path

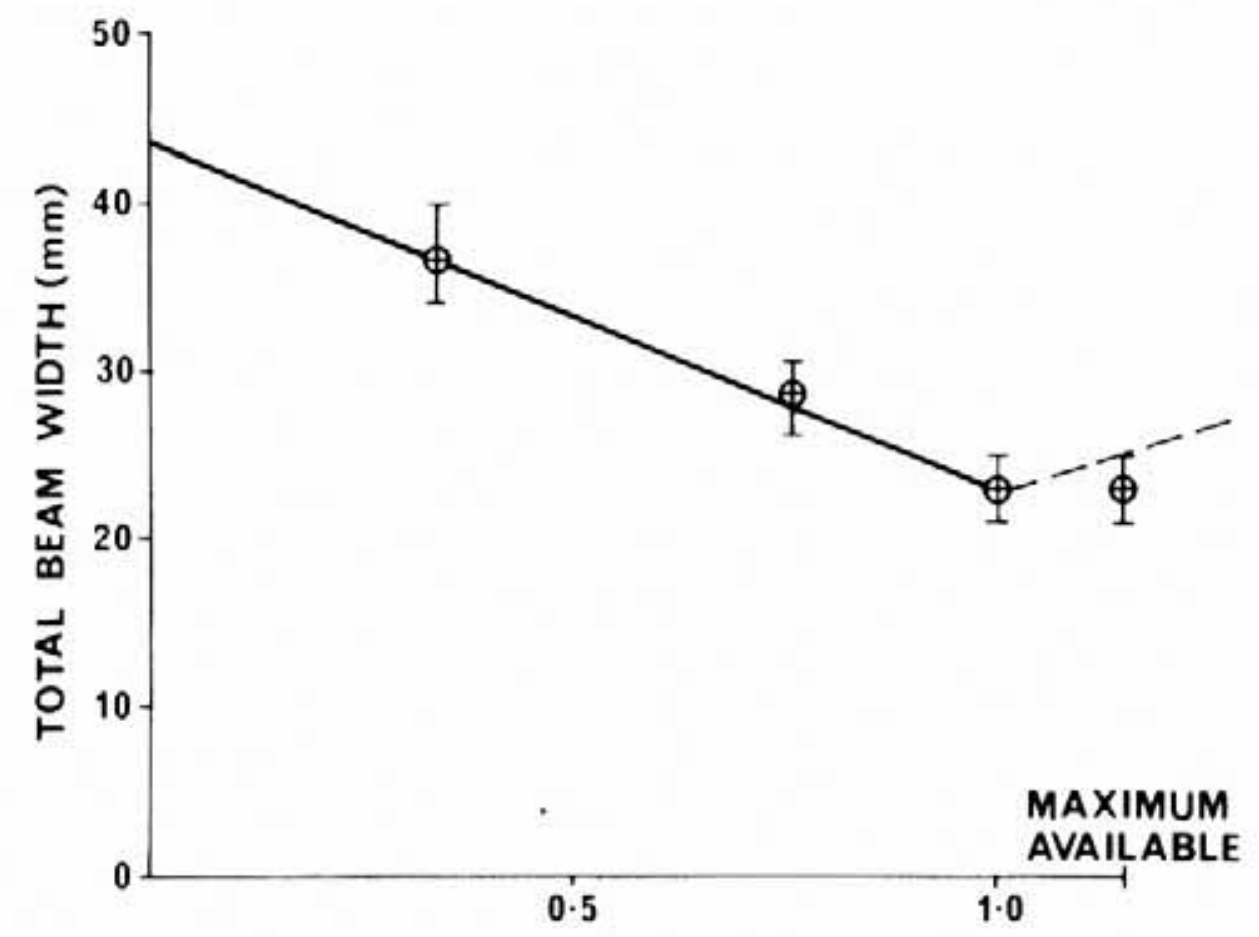

Fig. 8 Variation of beam width at injection with $r . f$. voltage in debuncher waveguide. 
the inflector. No change was detectable although a movement of +6.9 min would have been expected.

\section{CONCLUSIONS}

It may be concluded that the debuncher system behaves entirely as expected from the design considerations. The effect on the operation of the synchrotron has been beneficial, but, owing to the influence on the synchrotron beam of many other parameters than the energy spread in the injected beam, its effects may best be judged qualitatively rather than quantitatively.

The improvements may be 1 isted as follows :-

i) automatic fast energy correction of all injected electrons;

ii) reduced sensitivity of the synchrotron beam to variations in the linear accelerator which affect the energy spectrum and the energy stability during the pulse;

iii) improved capture of the injected beam.

The system has been in almost continuous use since it was first commissioned in the middle of 1970 . This report was given in abbreviated form (3) at the Chicago Conference, 1971.

\section{REFERENCES}

1. A. J. Lichtenberg, Reduction of energy spread at the output of a linac. CEA, Cambridge, Mass., U.S.A., Report CEA 22 (1957).

2. G. Saxon and I. White. Effect of Iris Spacing on the Performance of Electron Linear Accelerators. Proc. I.E.E. Vol. III, No. 3 (1964) 465.

3. M. C. Crowley-Milling and G. Saxon. The Use of a Debuncher in the Injection Path of an Electron Synchrotron. Trans. I.E.E.E. Nucl. Sci. NS18, No. 3 (1971), 968. 